\title{
Codon Usage of Autosomal Dominant Polycystic Kidney Disease Genes PKD1 and PKD2
}

\author{
Li Gun ${ }^{1}$, Tian Han ${ }^{2}$, Guo Rui ${ }^{3}$ \&Du Ning ${ }^{4}$ \\ ${ }^{1,2.3,4}$ Department of Biomedical Engineering, School of Electronics and Information Engineering, Xi'an \\ Technological University, Xi'an, Shaanxi Province, China
}

*Corresponding Author: Li Gun, Department of Biomedical Engineering, School of Electronics and Information Engineering, Xi'an Technological University, Xi'an, Shaanxi Province, China. E-mail: ligun@xatu.edu.cn

\begin{abstract}
:
Objective: To investigate the codon characteristics in autosomal dominant polycystic kidney disease (ADPKD) genes PKD1 and PKD2. Differences between PKD1 and PKD2 are theoretically compared and their potential applications are discussed.
\end{abstract}

Methods: Codon gene sequences of PKD1 and PKD2 are downloaded from the gene bank: http://www.ncbi.nlm.nih.gov and their codon characteristics are predicted via the codonW system. The results of relative synonymous codon usage $(R S C U)$ and $A 3 /(A 3+T 3) v s . G 3 /(G 3+C 3)$ et al are plotted and discussed.

Results: The codon usage preferences of PKD1 and PKD2 are affected by mutations, and less affected by the selectively effect. The RSCU value of CUG and GUG in PKD1 are greater than 2. The RSCU value of CUC, UCC, CAG, GAG and UGA are among 1.5 to 2, and others are less than 1.5. The codon usage preference in the PKD2 is not bias like it in the PKD1. All the RSCU values in PKD2 are less than 1.5.

Conclusions: The relationship between the $A 3 /(A 3+T 3)$ and $G 3 /(G 3+C 3)$ obey the laws of nature, $R S C U$ value of PKD1 is more than the value of PKD2 generally. All these information are important for studying the function of PKD1 and PKD2.

Keywords: Codon bias, PKD1, PKD2, ADPKD

\section{INTRODUCTION}

$A D P K D$ is associated with large interfamilial and intrafamilial variability [1]. Many scientists studied the mechanism [2-5] of the ADPKD and its treatment from various methods [6-8]. Form the genome perspective, there are two mutations have been found to be associated with adpkdPKD1 and PKD2 [9-12]. They are respectively on the 16th and 4th chromosomes [13-15]. Most of the ADPKD are caused by PKDI mutation. Most of the patients with ADPKD may require dialysis or kidney transplantation eventually [16-18]. Therefore, it is necessary to study the ADPKD from the perspective of genome. Many scientists such as Tao Yang, et al, explored the novel mutations of PKD1 gene in Chinese patients with ADPKD by targeted nextgeneration sequencing [19], Consugar, et al. studied the characteristics of large rearrangements in ADPKD and the PKD1/TSC2 contiguous gene syndrome [20]. Furthermore,
Pathogenicity [21], inactivation [22], development [23] of the PKD1 were also studied by many scientists. In this paper, based on the previous studies, the codon usage characteristics of PKD1 and PKD2 gene is cauculated via the CodonW, and analyzed via the PR2-bias plot analysis: A3 / (A3 + T3) vs. $\mathrm{G} 3 /(\mathrm{G} 3+\mathrm{C} 3)$. Then the RSCU values of PKD1 and PKD2 are plotted and discussed.

\section{Materials AND Methods}

Coding gene sequences of PKD1 and PKD2 are download from the website http://www.ncbi. nlm.nih.gov, and the accession numbers are: NC_000016.10:c2135898-2088708 and NC_00 0004.12:88007647-88077779. Then, the Codon $\mathrm{W}$ [24] is used to calculate the CAI, CBI, ENC and so on, the calculated results are further processed for getting the relative synonymous codon usage (RSCU), the PR2-bias plot analysis: A3 / (A3 + T3) vs. G3 / (G3 + C3), et al. 


\section{RESULTS AND DISCUSSION}

CodonW system is used to predict the codon usage characteristics of PKD1 and PKD2, and the overall results are shown in Table 1. in the table 1 Codon Adaptation Index (CAI) ranges from 0 to 1 , the greater the value, the stronger the sub-preference of the codon and the higher the gene expression level, the CAI value of PKD1 is 0.17 and in PKD2 is 0.18 respectively, they are about the same. But when the Codon Bias Index (CBI) is concerned, their values are 0.05 and -0.08 respectively in PKD1 and PKD2. The values of effective codon number (ENC) are about the same in them. But there is a clear distinction for the content of GC.

Table1. Nucleotide composition in the coding sequences of PKD1 and PKD2

\begin{tabular}{|c|c|c|c|c|c|c|c|c|c|c|c|}
\hline $\begin{array}{l}\text { Gene } \\
\text { name }\end{array}$ & $\begin{array}{c}\text { Acces } \\
\text { sion } \\
\text { numb } \\
\text { er }\end{array}$ & $\begin{array}{c}T \\
3 s\end{array}$ & $\begin{array}{l}\text { C } \\
\text { 3s }\end{array}$ & $\begin{array}{l}\text { A } \\
\text { 3s }\end{array}$ & $\begin{array}{l}\text { G } \\
3 \mathrm{~s}\end{array}$ & $\begin{array}{c}\text { C } \\
\text { A } \\
\text { I }\end{array}$ & $\begin{array}{c}\mathbf{C} \\
\text { BI }\end{array}$ & $\begin{array}{l}\mathbf{F} \\
\mathbf{o} \\
\mathbf{p}\end{array}$ & $\begin{array}{c}\mathbf{N} \\
\mathbf{c}\end{array}$ & $\begin{array}{l}\mathbf{G} \\
\mathbf{C} \\
3 \mathrm{~S}\end{array}$ & G \\
\hline PKD1 & $\begin{array}{l}\text { NC_0 } \\
00016 \\
.10\end{array}$ & $\begin{array}{l}0 . \\
2 \\
4\end{array}$ & $\begin{array}{l}0 . \\
3 \\
7\end{array}$ & $\begin{array}{l}0 . \\
1 \\
9\end{array}$ & $\begin{array}{l}0 . \\
3 \\
4\end{array}$ & $\begin{array}{l}0 . \\
1 \\
7\end{array}$ & $\begin{array}{l}0 . \\
05\end{array}$ & $\begin{array}{l}0 . \\
4 \\
3\end{array}$ & $\begin{array}{l}52 \\
.2 \\
4\end{array}$ & $\begin{array}{l}0 . \\
62\end{array}$ & $\begin{array}{l}0 \\
6 \\
4\end{array}$ \\
\hline PKD2 & $\begin{array}{l}\text { NC_0 } \\
00004 \\
.12\end{array}$ & $\begin{array}{l}0 . \\
3 \\
9\end{array}$ & $\begin{array}{l}0 . \\
2 \\
6\end{array}$ & $\begin{array}{l}0 . \\
3 \\
6\end{array}$ & $\begin{array}{l}0 . \\
2 \\
5\end{array}$ & $\begin{array}{l}0 . \\
1 \\
8\end{array}$ & $\begin{array}{l}- \\
0 . \\
08\end{array}$ & $\begin{array}{l}0 . \\
3 \\
7\end{array}$ & $\begin{array}{l}51 \\
.9 \\
5\end{array}$ & $\begin{array}{l}0 . \\
39\end{array}$ & $\dot{4}$ \\
\hline
\end{tabular}

The association between ENC and $\mathrm{GC}_{3}$ is shown in Figure 1. It can be seen that the PKD1 and PKD2 gene loci are distributed along the standard curve. The actual ENC values of KD1 and PKD2 genes are close to the expected ENC values, and locates of them are below the expected value. The codon usage preferences of PKD1 and PKD2 are affected by mutations, at the same time, the codon usage preferences is less affected by the selectively effect.

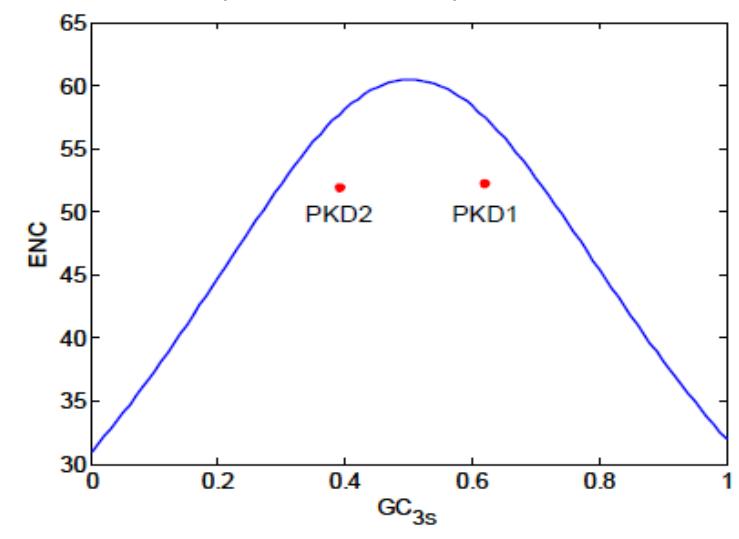

Figure1.Analysis of ENC and $G C_{3}$ relationship

If there is no mutation in the two complementary strands of DNA, the base content should obey the law $\mathrm{A}=\mathrm{T}$ and $\mathrm{G}=\mathrm{C}$. This method is usually used to analyze the PR2 bias occurring at the third place of the codon. The center of the graph follows the PR2 principle, $\mathrm{A}=\mathrm{T}$ and $\mathrm{G}=\mathrm{C}$. The distance between the center $(0.5,0.5)$ and the plot dot represents the degree and direction of the PR2 bias. From the Figure 2, it can be seen that the content of pyrimidine $(\mathrm{C}+\mathrm{T})$ at the third position of the hemoglobin codon gene is equal to the purine content $(A+G)$ in both PKD1 and PKD2.

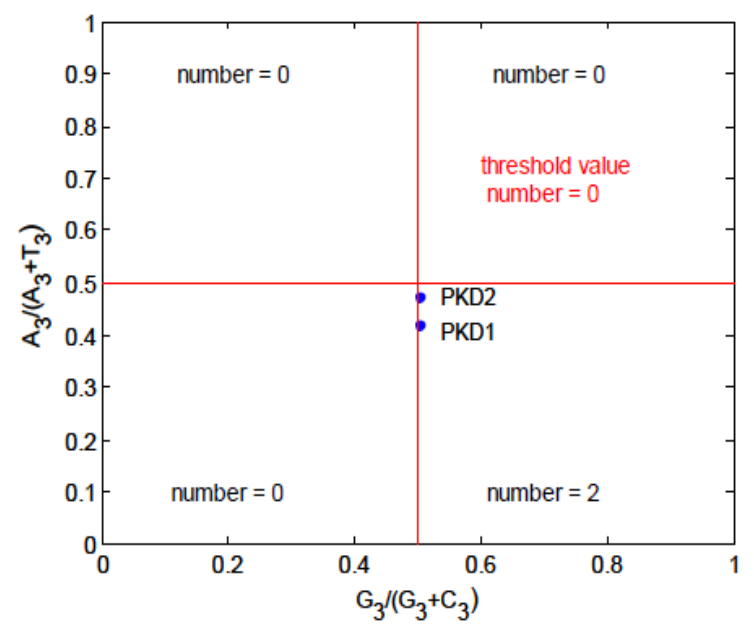

Figure2. PR2-bias plot analysis

Code preference is a common phenomenon in the evolutionary process. This phenomenon can not only reflect the evolution of biological groups, but also from the molecular level it can explain the basic phenomenon of biology, so it has very broad value of research and practical. The relative synonymous codon usage (RSCU) is an estimate of the preference for the use of synonymous codon, defined as the ratio of the observed value of the number of synonymous codons used to the expected value of occurrences of the codon. If the codon usage is not preferred, the RSCU value is 1 ; if a codon is used frequently, the RSCU value is greater than 1 ; if a codon usage frequency is low, the RSCU value is less than 1 . The RSCU value intuitively reflects the preference for codon usage in PKD1 (Figure 3) and PKD2 (Figure 4) is calculated. In the Figure 3, it can be seen that in the PKD1, the codon usage preference of CUG and GUG are greater than 2. The codon usage preference of CUC, UCC, CAG, GAG and UGA are among 1.5 to 2 . The codon usage preference others are less than 1.5. The value of RSCU is usually used as a measurement of the codon usage preference analysis. 

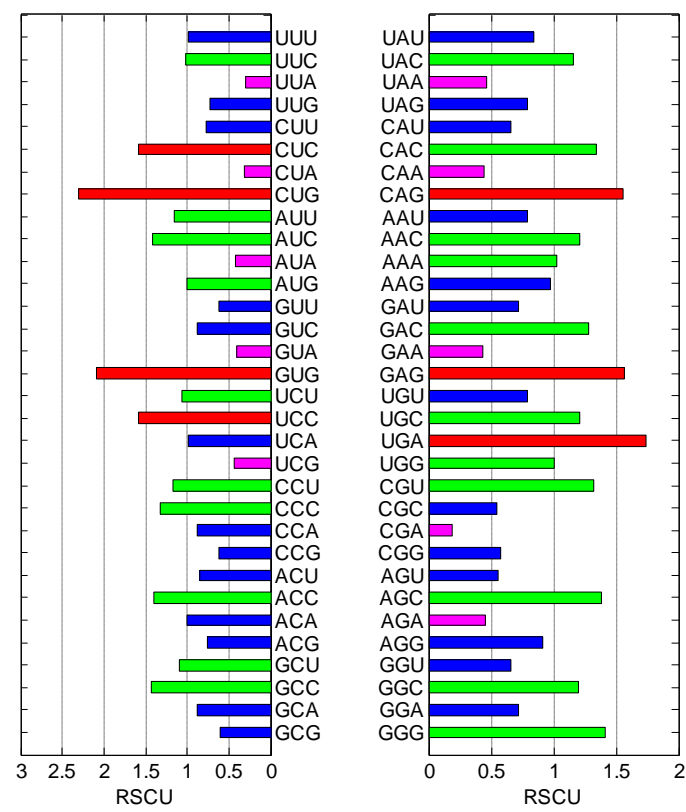

Figure3. RSCU of the PKD1 coding gene

In the Figure 3, it can be seen that the codon usage preference in the PKD2 is not bias like it in the PKD1. All the codon usage preferences are less then 1.5. And there are only five relative synonymous codon usage values less than 0.5 , see the pink bar in the figure 4. Correspondingly, there are ten relative synonymous codon usage values less than 0.5 . So the RSCU value in PKD2 is relatively more uniform and without greater deviation. Codons as a link between nucleic acids and proteins, it can be used to characterize the evolutionary relationships between genomes $[25,26]$. The closer the genetic relationship is, the closer the codon preference is, the closer the genetic relationship, and the less difference in codon preference [27]. When the ADPKD is concerned, people from different area may have nuances [28], for instance, Bunyong Phakdeekitcharoen studied the mutations of the replicated region of PKD1 in Asian population. So the codon usage is a reference for identification of porcine polycystic kidney disease [29, 30].

Although many scientists endeavor to study the mechanism of the ADPKD from the bioinformatics perspective [31-33], the development of the ADPKD is not very clear today. It is usually considered to be inherited from the parents, and affected by other factors such as toxin and infection. In the prevention of the disease, the genetic pathway is the best choice. This method is conducted in vitro fertilization firstly, and then uses single cells for genetic analysis. If the embryo is determined to be normal, then it can be re-implant the maternal uterus to continue pregnancy.
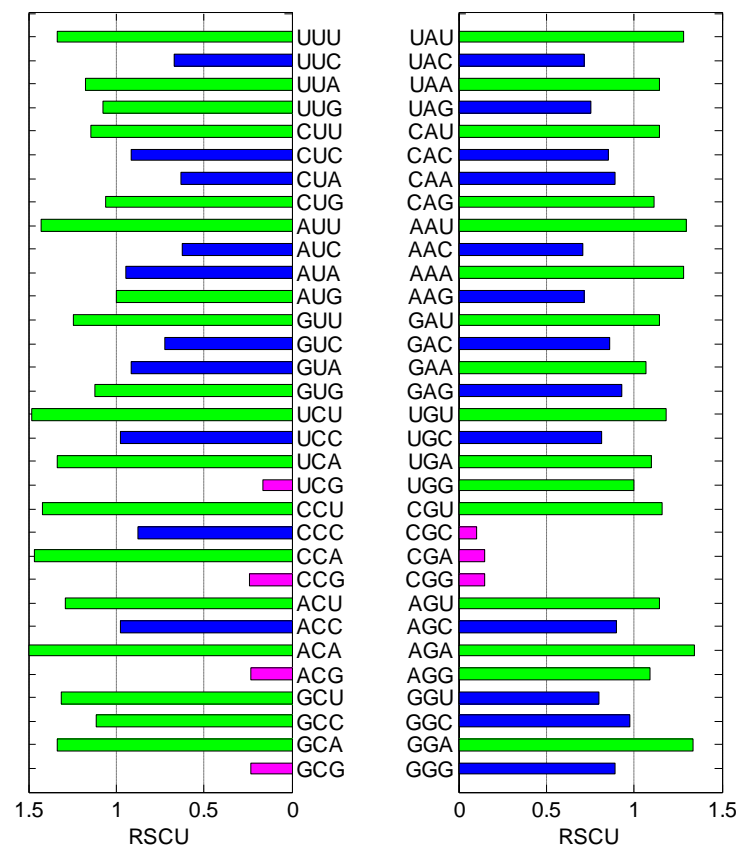

Figure4. $R S C U$ of the PKD2 coding gene

\section{CONCLUSION}

In the genetic engineering study, the optimal codon was identified by exploring the codon usage preference, measuring the level of expression of a protein coding gene, and finally constructing the gene expression vector based on the optimal codon to enhance the target gene number of expression, so as to improve the quality of production and has a certain practical value. In this paper, the preference of ADPKD related coding gene PKD1 and PKD2 are analyzed, such as the relationship between the $\mathrm{A} 3 /(\mathrm{A} 3+\mathrm{T} 3)$ and $\mathrm{G} 3 /(\mathrm{G} 3+\mathrm{C} 3)$, the relationship between $\mathrm{ENc}$ and $\mathrm{GC}_{3}$ are all studied and the RSCU of PKD1 and PKD2 coding gene is also discussed. The results show that codon usage preference value of PKD1 is more than the value of PKD2 generally. All these information are important for studying the function of PKD1 and PKD2.

\section{REFERENCES}

[1] Shatakshee Chatterjee, Srikant Prasad Verma, Priyanka Pandey. Profiling conserved biological pathways in Autosomal Dominant Polycystic Kidney Disorder (ADPKD) to elucidate key transcriptomic alterations regulating cystogenesis: A cross-species metaanalysis approach. Gene, 2017, 627(5): 434450

[2] Arlene B. Chapman. The importance of quantifying genetic heterogeneity in ADPKD. Kidney International, 2014, 85(2): 236-237 
[3] Binu M. Paul, Mark B. Consugar, Moonnoh Ryan Lee, Jamie L. Sundsbak, et al. Evidence of a third ADPKD locus is not supported by reanalysis of designated PKD3 families. Kidney International, 2014, 85(2): 383-392

[4] Xiaohong Li. Phosphorylation, protein kinases and ADPKD. Biochimica et Biophysica Acta (BBA) - Molecular Basis of Disease, 2011, 1812(10): 1219-1224

[5] Burhan M. Edrees, Mohammad Athar, Zainularifeen Abduljaleel, Faisal A Al-Allaf, et al. Functional alterations due to amino acid changes and evolutionary comparative analysis of ARPKD and ADPKD genes. Genomics Data, 2016, 10: 127-134

[6] Peter C. Harris, Sandro Rossetti. Determinants of Renal Disease Variability in ADPKD. Advances in Chronic Kidney Disease, 2010, 17(2): 131-139

[7] Masahiko Zuka, Tamehito Onoe, Mitsuhiro Kawano, Masakazu Yamagishi, Tohru Ohshima. Sudden death of a young male with previously undiagnosed autosomal dominant polycystic kidney disease (ADPKD). Legal Medicine, 2011, 13(1): 35-38

[8] Vicente E. Torres. Treatment Strategies and Clinical Trial Design in ADPKD. Advances in Chronic Kidney Disease, 2010, 17(2): 190-204

[9] Adam E. Mikolajczyk, Helen S. Te, Arlene B. Chapman. Gastrointestinal Manifestations of Autosomal-Dominant Polycystic Kidney Disease. Clinical Gastroenterology and Hepatology, 2017, 15(1): 17-24

[10] Tsukasa Nakamura, Eiichi Sato, Nobuharu Fujiwara, Yasuhiro Kawagoe, et al. Changes in Urinary Albumin Excretion, Inflammatory and Oxidative Stress Markers in ADPKD Patients with Hypertension. The American Journal of the Medical Sciences, 2012, 343(1): 46-51

[11] Hervé Husson, Partha Manavalan, Viatcheslav R. Akmaev, Ryan J. Russo, et al. New insights into ADPKD molecular pathways using combination of SAGE and microarray technologies. Genomics, 2004, 84(3): 497-510

[12] Gilberto Ka Kit Leung, Yiu Wah Fan. Chronic subdural haematoma and arachnoid cyst in autosomal dominant polycystic kidney disease (ADPKD). Journal of Clinical Neuroscience, 2005, 12(7): 817-819

[13] Yan-Kun Sha, Yan-Wei Sha, Li-Bin Mei, XianJing Huang, et al. Use of targeted sequence capture and high-throughput sequencing identifies a novel PKD1 mutation involved in adult polycystic kidney disease. Gene, 2017, 634: 1-4

[14] Kengo Hirota, Hiroyuki Akagawa, Hideaki Onda, Taku Yoneyama, et al. Association of Rare Non synonymous Variants in PKD1 and PKD2 with Familial Intracranial Aneurysms in a Japanese Population. Journal of Stroke and
Cerebrovascular Diseases, 2016, 25(12): 29002906

[15] Mikiko Soejima, Yasuo Sugita, Yoshiro Koda. An autopsy case of subarachnoid hemorrhage due to ruptured cerebral aneurysm associated with polycystic kidney disease caused by a novel PKD1 mutation. Forensic Science International, 2014, 242: e18-e21

[16] Melissa A. Cadnapaphornchai, Diana M. George, Amirali Masoumi, Kim McFann, et al. Effect of statin therapy on disease progression in pediatric ADPKD: Design and baseline characteristics of participants. Contemporary Clinical Trials, 2011, 32(3): 437-445

[17] Keiko Fukino, Junpei Ishiwata, Hiroki Shinohara, Tsukasa Oshima, et al. Noncompaction of the Ventricular Myocardium and Polycystic Kidney Disease: A Case Report. American Journal of Kidney Diseases, 2016, 67(6): 945-948

[18] Laura Rodriguez-Osorio, Maria Vanessa PerezGomez, Alberto Ortiz. Decreasing incidence of renal replacement therapy over time at the critical 50-59-year age range suggests a role for nephro protective therapy in ADPKD. Kidney International, 2015, 88(1): 193-194

[19] Tao Yang, Yan Meng, Xiaoming Wei, Jiandong Shen, et al. Identification of novel mutations of PKD1 gene in Chinese patients with autosomal dominant polycystic kidney disease by targeted next-generation sequencing. Clinica Chimica Acta, 2014, 433: 12-19

[20] Mark B. Consugar, Wai C. Wong, Patrick A. Lundquist, Sandro Rossetti, et al. Characterization of large rearrangements in autosomal dominant polycystic kidney disease and the PKD1/TSC2 contiguous gene syndrome. Kidney International, 2008, 74(11): 1468-1479

[21] Zishui Fang, Shiyan Xu, Yonghua Wang, Liwei Sun, et al. Pathogenicity analysis of novel variations in Chinese Han patients with polycystic kidney disease. Gene, 2017, 626: 433-441

[22] Kalani L. Raphael, Kevin A. Strait, Peter K. Stricklett, R. Lance Miller, et al. Inactivation of Pkd1 in principal cells causes a more severe cystic kidney disease than in intercalated cells. Kidney International, 2009, 75(6): 626-633

[23] Sandro Rossetti, Dominique Chauveau, Vickie Kubly, Jeffrey M Slezak, et al. Association of mutation position in polycystic kidney disease 1 (PKD1) gene and development of a vascular phenotype. The Lancet, 2003, 361(9376): 21962201

[24] Peden, J.F. CodonW. (PhD Thesis). University of Nottingham, Nottinghamshire. 1999

[25] Yang Liang, Mei He, Chun-Bo Teng. Evolution of the vesicular stomatitis viruses: Divergence 
and codon usage bias. Virus Research, 2014, 192: 46-51

[26] Laura Kubatko, Premal Shah, Radu Herbei, Michael A. Gilchrist. A codon model of nucleotide substitution with selection on synonymous codon usage. Molecular Phylogenetics and Evolution, 2016, 94: 290297

[27] Jasmine Saini, Uri Hershberg. B cell Variable genes have evolved their codon usage to focus the targeted patterns of somatic mutation on the complementarity determining regions. Molecular Immunology, 2015, 65(1): 157-167

[28] Bunyong Phakdeekitcharoen, Terry J. Watnick, Curie Ahn, Dae-Yeon Whang, et al. Thirteen novel mutations of the replicated region of PKD1 in an Asian population. Kidney International, 2000, 58(4): 1400-1412

[29] Jin He, Qingsong Wang, Jianhua Ye, Xiaoxiang $\mathrm{Hu}$, Ning Li. Identification of porcine polycystic kidney disease 1 (PKD1) gene: Molecular cloning, expression profile, and implication in disease model. Gene, 2011, 490(1-2): 37-46
[30] Ruth Thomas, Robert McConnell, Jo Whittacker, Peter Kirkpatrick, et al. Identification of Mutations in the Repeated Part of the Autosomal Dominant Polycystic Kidney Disease Type 1 Gene, PKD1, by Long-Range PCR. The American Journal of Human Genetics, 1999, 65(1): 39-49

[31] Fouad T. Chebib, Marie C. Hogan, Ziad M. ElZoghby, Maria V. Irazabal, et al. Autosomal Dominant Polycystic Kidney Patients May Be Predisposed to Various Cardio myopathies. Kidney International Reports, 2017, 2(5): 913923

[32] Jonathan Lai, Lopa Modi, Daryl Ramai, Matthew Tortora. Tuberous sclerosis complex and polycystic kidney disease contiguous gene syndrome with Moyamoya disease. Pathology Research and Practice, 2017, 213(4): 410-415

[33] Junya Yamamoto, Saori Nishio, Fumihiko Hattanda, Daigo Nakazawa, et al. Branchedchain amino acids enhance cyst development in autosomal dominant polycystic kidney disease. Kidney International, 2017, 92(2): 377-387

Citation: Li Gun, Tian Han, Guo Rui \& Du Ning. Codon Usage of Autosomal Dominant Polycystic Kidney Disease Genes PKD1 and PKD2. ARC Journal of Urology.2017;2(2):12-16. doi: dx.doi.org/10.20431/2456060X.0202002

Copyright: (c) 2017 Authors. This is an open-access article distributed under the terms of the Creative Commons Attribution License, which permits unrestricted use, distribution, and reproduction in any medium, provided the original author and source are credited. 